\title{
THE RELEVANCE OF FREIMAN'S THEOREM FOR COMBINATORIAL COMMUTATIVE ALGEBRA
}

\author{
JÜRGEN HERZOG, TAKAYUKI HIBI AND GUANGJUN ZHU*
}

\begin{abstract}
Freiman's theorem gives a lower bound for the cardinality of the doubling of a finite set in $\mathbb{R}^{n}$. In this paper we give an interpretation of his theorem for monomial ideals and their fiber cones. We call a quasi-equigenerated monomial ideal a Freiman ideal, if the set of its exponent vectors achieves Freiman's lower bound for its doubling. Algebraic characterizations of Freiman ideals are given, and finite simple graphs are classified whose edge ideals or matroidal ideals of its cycle matroids are Freiman ideals.
\end{abstract}

\section{INTRODUCTION}

Let $X$ be a finite subset of $\mathbb{Z}^{n}$, and let $A(X)$ be the affine hull of the set $X$, that is, the smallest affine subspace of $\mathbb{Q}^{n}$ containing $X$. The doubling of $X$ is the set $2 X=\{a+b: a, b \in X\}$. The starting point of this paper is the following celebrated theorem of Freiman [7]:

$$
|2 X| \geq(d+1)|X|-\left(\begin{array}{c}
d+1 \\
2
\end{array}\right),
$$

where $d$ is the dimension of the affine space $A(X)$.

Now let $K$ be a field and let $I$ be a graded ideal in the polynomial ring $S=$ $K\left[x_{1}, \ldots, x_{n}\right]$. We denote by $\mu(I)$ the minimal number of generators of $I$ and by $\ell(I)$ the analytic spread of $I$, that is, the Krull dimension of the fiber cone $F(I)=$ $\oplus_{k \geq 0} I^{k} / \mathfrak{m} I^{k}$. Here $\mathfrak{m}=\left(x_{1}, \ldots, x_{n}\right)$ is the graded maximal ideal of $S$. It has been noticed in [10, Theorem 1.9] that Freiman's theorem has an interesting consequence regarding the minimal number of generators of the square of a monomial ideal. Namely, it was shown that if $I \subset S$ is a monomial ideal with the property that all generators of $I$ have the same degree, then $\mu\left(I^{2}\right) \geq \ell(I) \mu(I)-\left(\begin{array}{c}\ell(I) \\ 2\end{array}\right)$.

For the application of Freiman's theorem to a monomial ideal $I \subset S$ and its fiber cone $F(I)$, it is sufficient to require that $I$ is quasi-equigenerated, by which we mean that the exponent vectors of all generators of $I$ lie in a hyperplane of $\mathbb{Z}^{n}$. This guarantees that the set of exponent vectors of the monomials $u \in G\left(I^{2}\right)$ is obtained from the set of exponent vectors of the monomials $v \in G(I)$ by doubling. Here, $G(I)$ denotes the unique minimal set of monomial generators of a monomial ideal $I$.

2010 Mathematics Subject Classification. Primary 13C99; Secondary 13A15, 13E15, 13H05, $13 \mathrm{H} 10$.

Key words and phrases. Monomial ideal, Freiman ideal, Freiman graph, Freiman matroid, fiber cone.

* Corresponding author. 
We call a quasi-equigenerated monomial ideal $I \subset S$ a Freiman ideal, if $\mu\left(I^{2}\right)=$ $\ell(I) \mu(I)-\left(\begin{array}{c}\ell(I) \\ 2\end{array}\right)$. Of course, $I$ is Freiman if and only if equality holds in (1) for the set $X$ of exponent vectors of the monomials $u \in G(I)$. The sets $X \subset \mathbb{Z}^{n}$ for which $|2 X|=(d+1)|X|-\left(\begin{array}{c}d+1 \\ 2\end{array}\right)$ are characterized by Stanescu in [19]. Here, in Theorem 1.3, we give several equivalent conditions for a quasi-equigenerated monomial ideal $I \subset S$ to be a Freiman ideal. For example, it is shown that $I$ is Freiman if and only if the fiber cone $F(I)$ of $I$ is Cohen-Macaulay and the defining ideal of $F(I)$ has a 2-linear resolution. This homological characterization of Freiman ideals can be deduced from the fact that for a Freiman ideal not only one has $\mu\left(I^{2}\right)=\ell(I) \mu(I)-\left(\begin{array}{c}\ell(I) \\ 2\end{array}\right)$, but also $\mu\left(I^{k}\right)=\left(\begin{array}{c}\ell+k-2 \\ k-1\end{array}\right) \mu(I)-(k-1)\left(\begin{array}{c}\ell+k-2 \\ k\end{array}\right)$ for all $k \geq 1$. Indeed, this fact is a consequence of a result of Böröczky, Santos and Serra [4, Corollary 7]. Now this formula for the minimal number of monomial generators of $I^{k}$ of a Freiman ideal $I$ easily yields that $h_{i}=0$ for all $i \geq 2$, where $\left(1, h_{1}, h_{2}, \ldots\right)$ is the $h$-vector of the fiber cone $F(I)$ of $I$. Then we apply a result of Eisenbud and Goto [5, Corollary 4.5] and obtain that $F(I)$ is normal. Since $F(I)$ is a toric ring, Hochster's theorem [14 finally yields that $F(I)$ is Cohen-Macaulay.

Even more surprising than the inequality $\mu\left(I^{2}\right) \geq \ell(I) \mu(I)-\left(\begin{array}{c}\ell(I) \\ 2\end{array}\right)$, and seemingly not noticed before in the study of toric rings, are the following consequences of the above mentioned results about the $h$-vector of the fiber cone $F(I)$ and of Freiman's theorem, which may phrased as follows: let $A$ be a standard graded toric $K$-algebra with $h$-vector $\left(1, h_{1}, h_{2}, \ldots\right)$. Then $h_{2} \geq 0$, and if $h_{2}=0$, then $h_{i}=0$ for all $i \geq 2$. The inequality $h_{2} \geq 0$ was first observed in [10, Corollary 2.6].

Being a Freiman ideal is a very restrictive condition. Therefore, one can expect that for natural classes of monomial ideals arising in combinatorial context a nice classification of Freiman ideals is possible. We illustrate this in two cases. In both cases we consider a finite simple graph $G$. We call $G$ a Freiman graph, if its edge ideal $I(G)$ is a Freiman ideal. This classification can be reduced to the case that $G$ is connected, see Corollary 2.2. In Theorem 2.3 it is shown that if $G$ is connected and $H$ is the subgraph of $G$ whose edges are the edges of all the 4-cycles of $G$, then $G$ is a Freiman graph if and only if there exist no primitive even walks in $G$, or otherwise $H$ is bipartite of type $(2, s)$ for some integer $s$, and there exist no primitive even walks in $G$ of length $>4$. The proof is inspired by the classification of edge ideals with 2-linear resolution, due to Ohsugi and Hibi [16]. In the special case that $G$ is bipartite this classification is even more explicit. Indeed, in Corollary 2.4 it is shown, that if $G$ is a connected bipartite graph, then $G$ is Freiman if and only if $G$ is a tree, or otherwise there exists a bipartite subgraph $H$ of $G$ of type $(2, s)$ for some integer $s$, a subset $S$ of $V(H)$ and for each $w \in S$ an induced tree $T_{w}$ of $G$ with $V(H) \cap V\left(T_{w}\right)=\{w\}$.

The other case studied here is that of the matroidal ideal $I_{M}$ of the cycle matroid $M$ of $G$. We call a matroid $M$ whose matroidal ideal $I_{M}$ is a Freiman ideal, a Freiman matroid. In Theorem 2.5 we show that the cycle matroid of $G$ is Freiman if and only if $G$ contains at most one cycle. It is a challenging open problem to classify all Freiman matroids. A few results in this direction, even for polymatroids, can be found in [11]. 
As an interesting consequence of the classification of the cycle matroids which are Freiman, it is shown in Theorem 2.13 that if $r$ denotes the regularity of the base ring of the cycle matroid of a graph $G$, then, unless the base ring is a polynomial ring, one has $3 \leq r \leq e$, where $e$ is the number of the edges of $G$.

\section{Freiman IDEALS AND THEIR FIBER CONES}

Let $K$ be a field and $S=K\left[x_{1}, \ldots, x_{n}\right]$ the polynomial ring in $n$ indeterminates over $K$, and let $I \subset S$ be a monomial ideal. The unique minimal set of monomial generators of $I$ will be denoted by $G(I)$. The number $\mu(I)=|G(I)|$ is the minimal number of generators of $I$. We write $\mathbf{x}^{\mathbf{c}}$ for the monomial $x_{1}^{c_{1}} \cdots x_{n}^{c_{n}}$ where $\mathbf{c}=$ $\left(c_{1}, \ldots, c_{n}\right)$.

Let $\mathbf{a}=\left(a_{1}, \ldots, a_{n}\right) \in \mathbb{Z}^{n}$ be an integer vector with all $a_{i}>0$. We say that $I$ is equigenerated (of degree $d$ ) with respect to a or simply say that $I$ is quasiequigenerated, if there exists an integer $d$ such that

$$
d=\langle\mathbf{a}, \mathbf{c}\rangle \text { for all } \mathbf{x}^{\mathbf{c}} \in G(I) \text {. }
$$

Here $\langle\mathbf{a}, \mathbf{c}\rangle=\sum_{i=1}^{n} a_{i} c_{i}$ denotes the standard scalar product of $\mathbf{a}$ and $\mathbf{c}$. The ideal $I$ is called equigenerated if $\mathbf{a}=(1,1, \ldots, 1)$.

The Krull dimension of the fiber cone $F(I)=\bigoplus_{k \geq 0} I^{k} / \mathfrak{m} I^{k}$ is called the analytic spread of $I$, and denoted $\ell(I)$. One always has

$$
\text { height } I \leq \ell(I) \leq \min \{\mu(I), n\} \text {. }
$$

Let $G(I)=\left\{u_{1}, \ldots, u_{m}\right\}$, and let $T=K\left[y_{1}, \ldots, y_{m}\right]$ be the polynomial ring over $K$ in the indeterminates $y_{1}, \ldots, y_{m}$. Consider the $K$-algebra homomorphism $\varphi: T \rightarrow$ $F(I)$ with $y_{j} \mapsto u_{j}+\mathfrak{m} I$ for $j=1, \ldots, m$. Then $F(I) \cong T / J$, where $J=\operatorname{Ker} \varphi$. Since $I$ is quasi-equigenerated, it follows that $F(I) \cong K\left[u_{1} t, \ldots u_{m} t\right] \subset T[t]$, where $t$ is a new indeterminate over $T$. Thus we see that $J$ is a toric prime ideal generated by binomials in $T$ which are homogeneous with respect to the standard grading of $T$.

The following theorem is a consequence of a famous theorem of Freiman [7] and its generalizations by Böröczky et al [4].

Theorem 1.1. Let $I \subset S$ be a quasi-equigenerated monomial ideal whose analytic spread is $\ell(I)$. Then

$$
\mu\left(I^{k}\right) \geq\left(\begin{array}{c}
\ell(I)+k-2 \\
k-1
\end{array}\right) \mu(I)-(k-1)\left(\begin{array}{c}
\ell(I)+k-2 \\
k
\end{array}\right)
$$

for all $k \geq 1$.

Proof. Let $S(I) \subset \mathbb{Z}^{n}$ be the set of exponent vectors of the elements of $G(I)$. Since $I$ is a quasi-equigenerated monomial ideal, $I^{k}$ are quasi-equigenerated monomial ideals for all $k \geq 1$. It follows that $\mu\left(I^{k}\right)=\left|S\left(I^{k}\right)\right|=|k S(I)|$ for all $k \geq 1$. Let $d$ be the 
Freiman dimension of $S(I)$, then from [4, Corollary 2],

$$
\begin{aligned}
|k S(I)| & \geq\left(\begin{array}{c}
d+k-1 \\
k-1
\end{array}\right)|S(I)|-(k-1)\left(\begin{array}{c}
d+k-1 \\
k
\end{array}\right) \\
& =\left(\begin{array}{c}
d+k-1 \\
k-1
\end{array}\right) \mu(I)-(k-1)\left(\begin{array}{c}
d+k-1 \\
k
\end{array}\right) .
\end{aligned}
$$

On the other hand, by [10, Theorem 1.9] one has $\ell(I)=d+1$. Thus the assertion follows.

The above theorem implies in particular that $\mu\left(I^{2}\right) \geq l(I) \mu(I)-\left(\begin{array}{c}l(I) \\ 2\end{array}\right)$. In [11], the first and third author of this paper called an equigenerated monomial ideal a Freiman ideal, if equality holds. Here we extend this definition and call a quasi-equigenerated ideal $I$ a Freiman ideal, if

$$
\mu\left(I^{2}\right)=l(I) \mu(I)-\left(\begin{array}{c}
l(I) \\
2
\end{array}\right) .
$$

In the next theorem we will give some characterizations of Freiman ideals in terms of the Hilbert series of their fiber cones. To this end we recall some concepts from commutative algebra.

Let $R$ be a standard graded $K$-algebra. We denote its Krull dimension by $\operatorname{dim} R$ and its embedding dimension by emb $\operatorname{dim} R$. The Hilbert series $\operatorname{Hilb}_{R}(t)=$ $\sum_{k \geq 0} \operatorname{dim}_{K} R_{k} t^{k}$ of $R$ is of the form

$$
\operatorname{Hilb}_{R}(t)=h(R ; t) /(1-t)^{\operatorname{dim} R},
$$

where $h(R ; t)=1+h_{1} t+h_{2} t^{2}+\cdots$ is a polynomial with $h_{1}=\operatorname{emb} \operatorname{dim} R-\operatorname{dim} R$. The polynomial $h(R ; t)$ is called the $h$-polynomial of $R$, and the finite coefficient vector of $h(R ; t)$ is called the $h$-vector of $R$.

The multiplicity of $R$ is defined to be $e(R)=\sum_{i \geq 0} h_{i}$. By Abhyankar [1] it is known that

$$
\operatorname{emb} \operatorname{dim} R \leq e(R)+\operatorname{dim} R-1,
$$

if $R$ is a domain. The same inequality holds if $R$ is Cohen-Macaulay [17]. The $K$-algebra $R$ is said to have minimal multiplicity if emb $\operatorname{dim}(R)=e(R)+\operatorname{dim} R-1$.

We first observe

Corollary 1.2. Let $I \subset S$ be a quasi-equigenerated monomial ideal with analytic spread $\ell$, and let $\left(1, h_{1}, h_{2}, \ldots\right)$ be the $h$-vector of the fiber cone $F(I)$ of $I$. Then

$$
\sum_{i=2}^{k}\left(\begin{array}{c}
\ell+k-i-1 \\
k-i
\end{array}\right) h_{i} \geq 0 \text { for all } k \geq 2 \text {. }
$$

In particular, $h_{2} \geq 0$.

Proof. The Hilbert series of the fiber cone $F(I)$ of $I$ is

$$
\operatorname{Hilb}_{F(I)}(t)=\sum_{k \geq 0} \mu\left(I^{k}\right) t^{k}=\frac{1+h_{1} t+h_{2} t^{2}+h_{3} t^{3}+h_{4} t^{4}+\cdots}{(1-t)^{\ell}}
$$




$$
\begin{aligned}
& =\left(1+h_{1} t+h_{2} t^{2}+\cdots\right)\left(1+\ell t+\left(\begin{array}{c}
\ell+1 \\
2
\end{array}\right) t^{2}+\left(\begin{array}{c}
\ell+2 \\
3
\end{array}\right) t^{3}+\cdots\right) \\
& =1+\left(h_{1}+\ell\right) t+\left(h_{1} \ell+\left(\begin{array}{c}
\ell+1 \\
2
\end{array}\right)+h_{2}\right) t^{2}+\cdots \\
& +\left[\left(\begin{array}{c}
\ell+k-1 \\
k
\end{array}\right)+\sum_{i=1}^{k}\left(\begin{array}{c}
\ell+k-i-1 \\
k-i
\end{array}\right) h_{i}\right] t^{k}+\cdots .
\end{aligned}
$$

It follows that

$$
\mu(I)=h_{1}+\ell, \text { and } \mu\left(I^{2}\right)=h_{1} \ell+\left(\begin{array}{c}
\ell+1 \\
2
\end{array}\right)+h_{2}=\ell \mu(I)-\left(\begin{array}{l}
\ell \\
2
\end{array}\right)+h_{2} .
$$

Moreover, for all $k \geq 3$ we have

$$
\begin{aligned}
\mu\left(I^{k}\right) & =\left(\begin{array}{c}
\ell+k-1 \\
k
\end{array}\right)+\left(\begin{array}{c}
\ell+k-2 \\
k-1
\end{array}\right) h_{1}+\left(\begin{array}{c}
\ell+k-3 \\
k-2
\end{array}\right) h_{2}+\cdots+\left(\begin{array}{l}
\ell \\
1
\end{array}\right) h_{k-1}+h_{k} \\
& =\left(\begin{array}{c}
\ell+k-1 \\
k
\end{array}\right)+\left(\begin{array}{c}
\ell+k-2 \\
k-1
\end{array}\right)(\mu(I)-\ell)+\sum_{i=2}^{k}\left(\begin{array}{c}
\ell+k-i-1 \\
k-i
\end{array}\right) h_{i} \\
& =\left(\begin{array}{c}
\ell+k-2 \\
k-1
\end{array}\right) \mu(I)-(k-1)\left(\begin{array}{c}
\ell+k-2 \\
k
\end{array}\right)+\sum_{i=2}^{k}\left(\begin{array}{c}
\ell+k-i-1 \\
k-i
\end{array}\right) h_{i} .
\end{aligned}
$$

Therefore, Theorem 1.1 implies that $h_{2} \geq 0$ and $\sum_{i=2}^{k}\left(\begin{array}{c}\ell+k-i-1 \\ k-i\end{array}\right) h_{i} \geq 0$ for all $k \geq 2$.

Let $I$ be a graded ideal. An ideal $J \subseteq I$ is called a reduction of $I$ if $I^{k+1}=J I^{k}$ for some nonnegative integer $k$. The reduction number of $I$ with respect to $J$ is defined to be

$$
r_{J}(I)=\min \left\{k \mid I^{k+1}=J I^{k}\right\} .
$$

A reduction $J$ of $I$ is called a minimal reduction if it does not properly contain any other reduction of $I$. If $I$ is equigenerated and $|K|=\infty$, then a graded minimal reduction of $I$ exists. In this case the reduction number of $I$ is defined to be the number

$$
r(I)=\min \left\{r_{J}(I) \mid J \text { is a minimal reduction of } I\right\} .
$$

Now we are ready to present the main result of this section.

Theorem 1.3. Let $I \subset S$ be a quasi-equigenerated monomial ideal with analytic spread $\ell$, and let $\left(1, h_{1}, h_{2}, \ldots\right)$ be the h-vector of the fiber cone $F(I)$ of $I$. Write $F(I)=T / J$, where $T$ is a polynomial ring over $K$, and $J$ is contained in the square of the graded maximal ideal of $T$. Then the following conditions are equivalent:

(a) I is a Freiman ideal.

(b) $\mu\left(I^{k}\right)=\left(\begin{array}{c}\ell+k-2 \\ k-1\end{array}\right) \mu(I)-(k-1)\left(\begin{array}{c}\ell+k-2 \\ k\end{array}\right)$ for all $k \geq 1$.

(c) $\mu\left(I^{k}\right)=\left(\begin{array}{c}\ell+k-2 \\ k-1\end{array}\right) \mu(I)-(k-1)\left(\begin{array}{c}\ell+k-2 \\ k\end{array}\right)$ for some $k \geq 2$.

(d) $h_{2}=0$. 
(e) $h_{i}=0$ for all $i \geq 2$.

(f) $F(I)$ has minimal multiplicity.

(g) $F(I)$ is Cohen-Macaulay and the defining ideal of $F(I)$ has a 2-linear free T-resolution.

Moreover, if $|K|=\infty$, then the above conditions are equivalent to

(h) $F(I)$ is Cohen-Macaulay and $r(I)=1$.

Proof. (a) $\Rightarrow$ (b) follows from [4, Corollary 7], (b) $\Rightarrow$ (c) is trivial, and (c) $\Rightarrow$ (b) also follows from [4, Corollary 7].

In the proof of Corollary 1.2, we have seen that $\mu(I)=h_{1}+\ell, \mu\left(I^{2}\right)=\ell \mu(I)-$ $\left(\begin{array}{l}\ell \\ 2\end{array}\right)+h_{2}$ and

$$
\mu\left(I^{k}\right)=\left(\begin{array}{c}
\ell+k-2 \\
k-1
\end{array}\right) \mu(I)-(k-1)\left(\begin{array}{c}
\ell+k-2 \\
k
\end{array}\right)+\sum_{i=2}^{k}\left(\begin{array}{c}
\ell+k-i-1 \\
k-i
\end{array}\right) h_{i} .
$$

By definition, $I$ is a Freiman ideal, if and only if $\mu\left(I^{2}\right)=\ell \mu(I)-\left(\begin{array}{l}\ell \\ 2\end{array}\right)$. This is equivalent to saying that $h_{2}=0$. This proves (a) $\Longleftrightarrow(\mathrm{d})$.

(b) $\Longleftrightarrow$ (e) follows from [11, Proposition 1.8 (b)].

(e) $\Rightarrow(\mathrm{f})$ : Notice that $e(R)=\sum_{i \geq 0} h_{i}$. If $h_{i}=0$ for $i \geq 2$, then

$$
e(R)=h_{0}+h_{1}=1+h_{1}=1+\mu(I)-\ell .
$$

This means that $F(I)$ has minimal multiplicity.

(f) $\Rightarrow(\mathrm{g})$ : After a base field extension, we may assume that $K$ is algebraically closed. Since $F(I)$ has minimal multiplicity, it follows that $F(I)$ is normal by $[5$, Corollary 4.5]. Since $F(I)$ is a toric ring, Hochster's theorem [14] implies that $F(I)$ is Cohen-Macaulay and $h_{i}=0$ for all $i \geq 2$. Thus from [11, Corollary 1.5] we obtain that the defining ideal of $F(I)$ has a 2-linear free $T$-resolution.

$(\mathrm{g}) \Rightarrow(\mathrm{d})$ : We may assume that $K$ is infinite. Since $F(I)$ is Cohen-Macaulay we can choose a regular sequence $t_{1}, \ldots, t_{\ell}$ of linear forms of $T_{1}$ which is also regular on $F(I)$. We let $\bar{T}=T /\left(t_{1}, \ldots, t_{\ell}\right)$ and denote by $\bar{J}$ the image of $J$ in $\bar{T}$. Since $t_{1}, \ldots, t_{\ell}$ is a regular sequence on $T / J$ and $J$ has a 2-linear resolution, it follows that $\operatorname{dim} \bar{T} / \bar{J}=0$ and $\bar{J}$ has a 2-linear resolution. It follows that $\bar{J}=\mathfrak{m}_{\bar{T}}^{2}$, where $\mathfrak{m}_{\bar{T}}^{2}$ is the graded maximal ideal of $\bar{T}$, since the only $\mathfrak{m}_{\bar{T}}$-primary ideals with linear resolution are powers of the maximal ideal. Thus we see that $\overline{F(I)}_{i}=0$ for $i \geq 2$, where $\overline{F(I)}=F(I) /\left(y_{1}, \ldots, y_{\ell}\right)$, and $y_{i}=t_{i}+J$ for $i=1, \ldots, \ell$. Since $y_{1}, \ldots, y_{\ell}$ is a regular sequence of linear form, the $h$-vector of $F(I)$ and that of $\overline{F(I)}$ coincide. Hence the conclusion follows.

$(\mathrm{g}) \Rightarrow(\mathrm{h})$ : We assume that $K$ is infinite, and let $y_{1}, \ldots, y_{\ell}$ be as in the proof $(\mathrm{g}) \Rightarrow$ (d). We write $y_{i}=f_{i}+\mathfrak{m} I$ with $f_{i} \in I$ for $i=1, \ldots, \ell$, and let $J=\left(f_{1}, \ldots, f_{\ell}\right)$. Now $\overline{F(I)}_{i}=0$ for $i \geq 2$ is equivalent to saying that $I^{2}=J I+\mathfrak{m} I^{2}$. Thus, Nakayama's lemma yields $I^{2}=J I$, as desired.

$(\mathrm{h}) \Rightarrow(\mathrm{d})$ : Since $I$ is quasi-equigenerated, $K$ is infinite and $F(I)$ is CohenMacaulay, a reduction ideal $J$ of $I$ with $r_{J}(I)=1$ can be chosen of the form $J=$ $\left(f_{1}, \ldots, f_{\ell}\right)$ such that the sequence $y_{i}=f_{i}+\mathfrak{m} I$ with $i=1, \ldots, \ell$ is a regular sequence on $F(I)$. Since $I^{2}=J I$ it follows that $\overline{F(I)_{i}}=0$ for $i \geq 2$, where 
$\overline{F(I)}=F(I) /\left(y_{1}, \ldots, y_{\ell}\right)$. Since $h$-vector of $F(I)$ and that of $\overline{F(I)}$ coincide, the assertion follows.

\section{Classes of Freiman ideals}

2.1. Freiman graphs. Let $K$ be a field and $G$ be a finite simple graph on $[n]$. The ideal $I(G) \subset S=K\left[x_{1}, \ldots, x_{n}\right]$ generated by the monomials $x_{i} x_{j}$ with $\{i, j\} \in E(G)$ is called the edge ideal of $G$. We say that $G$ is a Freiman graph, if $I(G)$ is a Freiman ideal. In this subsection we want to classify all Freiman graphs. Notice that $F(I(G))$ is isomorphic to the edge ring $K[G]=K\left[x_{i} x_{j}:\{i, j\} \in E(G)\right]$ of $G$. Let $T=K\left[z_{e}: e \in E(G)\right]$ be the polynomial ring in the indeterminates $z_{e}$, and let $I_{G}$ be the kernel of the $K$-algebra homomorphism $T \rightarrow K[G]$ with $z_{e} \mapsto x_{i} x_{j}$ for $e=\{i, j\}$. Then $K[G] \cong T / I_{G}$.

Certainly $G$ is Freiman, if $K[G]$ is the polynomial ring. The monomials $x_{i} x_{j}$ with $\{i, j\} \in E(G)$ are algebraic independent if and only if $K[G]$ is a polynomial ring, which is the case if and only if $I_{G}=0$.

In order to describe the generators of $I_{G}$ one has to consider walks in $G$. A walk $W$ of length $r$ of $G$ is a sequence of vertices $i_{0}, i_{1}, \ldots, i_{r}$ such that $\left\{i_{j}, i_{j+1}\right\}$ is an edge of $G$ for $j=0, \ldots, r-1$. If $i_{0}=i_{r}$, then $W$ is called a closed walk. The closed walk is called even (odd), if $r$ is even (odd). It is called a cycle, if the vertices $i_{j}$ with $0 \leq j \leq r-1$ are pairwise distinct.

Now we have

Proposition 2.1. The edge ring $K[G]$ of $G$ is a polynomial ring, if and only if each connected component of $G$ contains at most one cycle, and this cycle is odd.

Proof. Let $G_{1}, \ldots, G_{s}$ be the connected components of $G$. Then $K[G]=\bigotimes_{i=1}^{s} K\left[G_{i}\right]$. Thus $K[G]$ is a polynomial ring if and only if each $K\left[G_{i}\right]$ is a polynomial ring. Hence we may assume that $G$ is connected and have to show that $K[G]$ is a polynomial ring if and only if $G$ contains at most one cycle, and this cycle is odd.

Let $W: i_{0}, i_{1}, \ldots, i_{2 s}$ be an even closed walk, and set $e_{j}=\left\{i_{j}, i_{j+1}\right\}$ for $j=$ $0, \ldots, 2 s-1$. Then

$$
f_{W}=\prod_{k=0}^{s-1} z_{e_{2 k}}-\prod_{k=0}^{s-1} z_{e_{2 k+1}} \in I_{G},
$$

and all binomial generators of $I_{G}$ are of this form, see [16, Lemma 1.1]. Thus $K[G]$ is a polynomial ring if and only if $f_{W}=0$ for each even closed walk in $G$.

Suppose that $K[G]$ is a polynomial ring and $G$ contains an even cycle $C$. Then $f_{C} \neq 0$, a contradiction. Therefore, $G$ contains no even cycles. Suppose $G$ contains at least two odd cycles, say $C_{1}$ and $C_{2}$. Let $i$ be a vertex of $C_{1}$ and $j$ be a vertex of $C_{2}$. Then, since $G$ is connected, there exists a walk $W$ connecting $i$ with $j$. Now we consider the following even closed walk $W^{\prime}$ : we first walk around $C_{1}$ starting with $i$. Then we walk along $W$ from $i$ to $j$. Then we continue our walk around $C_{2}$ to come back to $j$. From there we walk back to $i$ along $W$, but backwards. Obviously, $f_{W^{\prime}} \neq 0$. Thus there cannot be two odd cycles. This proves one direction of the proposition. 
Conversely, suppose that $G$ contains only one odd cycle, it is enough to prove that $I_{G}=0$. By [16, Lemma 3.1 and Lemma 3.2], the toric ideal $I_{G}$ is generated by the binomials $f_{W}$, where $W$ is an even closed walk of $G$ of one of the following types: (1) $W$ is an even cycle of $G$; (2) $W=\left(C_{1}, C_{2}\right)$, where $C_{1}$ and $C_{2}$ are odd cycles of $G$ having exactly one common vertex; (3) $W=\left(C_{1}, W_{1}, C_{2}, W_{2}\right)$, where $C_{1}$ and $C_{2}$ are odd cycles of $G$ having no common vertex and where $W_{1}$ and $W_{2}$ are walks of $G$ both of which combine a vertex $v_{1}$ of $C_{1}$ and a vertex $v_{2}$ of $C_{2}$. Walks of these three types are called primitive.

By the hypothesis that $G$ contains only one odd cycle, there cannot exist primitive even closed walks of $G$. Therefore, $I_{G}=0$.

Corollary 2.2. Let $G$ be a finite simple graph with $r$ connected components. Then following conditions are equivalent:

(a) $G$ is a Freiman graph.

(b) All connected components of $G$ are Freiman graphs and at least $r-1$ of its connected components contain at most one cycle, and this cycle is odd.

Proof. Let $G_{1}, \ldots, G_{r}$ be the connected components of $G$, and let $\operatorname{Hilb}_{K\left[G_{i}\right]}(t)=$ $h\left(K\left[G_{i}\right] ; t\right) /(1-t)^{d_{i}}$ with $d_{i}=\operatorname{dim} K\left[G_{i}\right]$. Then, since $K[G]=\bigotimes_{i=1}^{r} K\left[G_{i}\right]$, it follows that

$$
\operatorname{Hilb}_{K[G]}(t)=\prod_{i=1}^{r} \operatorname{Hilb}_{K\left[G_{i}\right]}(t)=h(K[G] ; t) /(1-t)^{d},
$$

where $h(K[G] ; t)=\prod_{i=1}^{r} h\left(K\left[G_{i}\right] ; t\right)$ and $d=\sum_{i=1}^{r} d_{i}=\operatorname{dim} K[G]$.

$(\mathrm{a}) \Rightarrow(\mathrm{b})$ : Since $G$ is Freiman, Theorem $[1.3$ implies that $\operatorname{deg} h(K[G] ; t) \leq 1$. Therefore, $\operatorname{deg} h\left(K\left[G_{i}\right] ; t\right) \leq 1$ with equality for at most one $i$. Thus (b) follows from Proposition 2.1 together with Theorem 1.3 .

(b) $\Rightarrow(\mathrm{a})$ : The assumptions in (b) together with Theorem 1.3 and Proposition 2.1 imply that $\operatorname{deg} h\left(K\left[G_{i}\right] ; t\right) \leq 1$ and $\operatorname{deg} h\left(K\left[G_{i}\right] ; t\right)=0$ for all $h\left(K\left[G_{i}\right] ; t\right)$ but possibly one of them. Thus $\operatorname{deg} h(K[G] ; t) \leq 1$, which by Theorem 1.3 implies that $G$ is Freiman.

Due to Corollary 2.2 it is enough to consider only connected graphs for the classification of Freiman graphs.

Theorem 2.3. Let $G$ be a finite simple connected graph, and let $H$ be the subgraph of $G$ whose edges are the edges of all the 4-cycles of $G$. Then $G$ is Freiman, if and only if there exist no primitive even walks in $G$, or otherwise $H$ is bipartite of type $(2, s)$ for some integer $s$, and there exist no primitive even walks in $G$ of length $>4$.

Proof. If $G$ has no primitive even walks, then $K[G]$ is the polynomial ring, and hence $G$ is Freiman. Suppose now that $H$ is bipartite of type $(2, s)$ for some $s$, and there exist no primitive even walks in $G$ of length $>4$. Since $G$ admits no primitive even walks of length $>4$, the ideal $I_{G}$ is generated by the binomials $f_{C}$ with $C$ a 4 -cycle of $G$. In particular, $I_{G}=I_{H}$, and $K[G]$ is a polynomial extension of $K[H]$. Since $H$ is bipartite of type $(2, s)$, the ideal $I_{H}$ may be viewed as the ideal of 2 -minors of a 
$2 \times s$-matrix of indeterminates. Hence the Eagon-Northcott complex [6] provides a 2-linear resolution of $I_{H}\left(=I_{G}\right)$. By Theorem 1.3, this implies that $G$ is Freiman.

Conversely, suppose that $G$ is Freiman. Then Theorem 1.3 implies that $I_{G}$ has no generators of degree $>2$, and hence $G$ admits no primitive even walks of length $>4$. We may assume that $H$ is bipartite of type $(r, s)$ with $r \leq s$. Since the edge set of $H$ is the union of the edges of the 4-cycles of $G$, we must have $r \geq 2$. Also it follows from this fact that $H$ is a complete bipartite graph if $r=2$. Indeed, suppose that one edge is missing, say the edge $\{2, r\}$. Then $\{1, r\} \in E(H)$ and $\{1, r\}$ belongs to a 4-cycle. Any 4-cycle containing the edge $\{1, r\}$ is of the form $r, 1, i, 2, r$ for some $i \neq r$. Hence $\{2, r\} \in E(H)$, a contradiction.

It remains to be show that $r=2$. Suppose $r>2$, and let $\left\{v_{1}, \ldots, v_{r}\right\} \cup$ $\left\{w_{1}, \ldots, w_{s}\right\}$ be the bipartition of the graph $H$. Since $H$ is the union of the edges of the 4-cycles of $G$, we may assume that, after a relabeling of the vertices, $C_{1}: v_{1}, w_{1}, v_{2}, w_{2}, v_{1}$ is a 4 -cycle of $H$. Since $r>2$, there must exist another 4-cycle $C_{2}$ of $H$ which contains the vertex $v_{3}$. Then $C_{1}$ and $C_{2}$ have only one vertex or one edge in common, or the cycles $C_{1}$ and $C_{2}$ are disjoint. But in the last case there is a walk in $G$ connecting a vertex of $C_{1}$ with a vertex of $C_{2}$ since $G$ is connected. In any case we have in this situation a primitive even walk of length $>4$, a contradiction.

Corollary 2.4. Let $G$ be a finite connected bipartite graph. Then $G$ is Freiman if and only if $G$ is a tree, or there exists a bipartite subgraph $H$ of $G$ of type $(2, s)$, a subset $S$ of $V(H)$ and for each $w \in S$ an induced tree $T_{w}$ of $G$ with $V(H) \cap V\left(T_{w}\right)=$ $\{w\}$.

Proof. If $G$ is a tree or if there exists a bipartite subgraph $H$ of $G$ satisfying the conditions of the corollary, then the subgraph $H$ is the union of all 4-cycles of $G$, and moreover there exist no primitive even walks in $G$ of length $>4$. Therefore, $G$ is Freiman, by Theorem 2.3 .

Conversely, assume that $G$ is Freiman and let $H$ be as defined in Theorem 2.3. Then Theorem 2.3 implies that $H=\emptyset$ or $H$ is bipartite of type $(2, s)$ for some $s$. If $H=\emptyset$, then Theorem 2.3 implies that $G$ has no even cycles of any length. Since $G$ is bipartite and connected, this implies that $G$ is a tree.

Suppose now that $H$ is bipartite of type $(2, s)$. If $G=H$, then there is nothing to prove. Otherwise there exists $v \in V(G) \backslash V(H)$. Since $G$ is connected there exists a walk $P_{1}$ outside of $H$ connecting $v$ with $w_{1} \in V(H)$. Assume there is another walk $P_{2}$ outside $H$ connecting $v$ with $w_{2} \in V(H)$ with $w_{1} \neq w_{2}$. Then there exists $w$ belonging to $P_{1}$ and $P_{2}$ such that the subwalk of $P_{1}$ connecting $w$ with $w_{1}$ and the subwalk of $P_{2}$ connecting $w$ with $w_{2}$ have only the vertex $w$ in common. Then we get a cycle walking from $w_{1}$ to $w$ to $w_{2}$, and from $w_{2}$ back to $w_{1}$ (inside $H$ ). Since $G$ is bipartite, this cycle must be even, a contradiction because by Theorem 2.3 . $G$ has even cycles of length at most 4 . But all even cycles of length 4 belong to $H$. It follows that each vertex $v$ of $G$ not belonging to $H$ is connected with at most one vertex $w \in H$.

Now let $w \in H$, and let $V$ be the set of vertices $v \in V(G) \backslash V(H)$ which are connected to $w$ via a walk. We denote by $T_{w}$ the restriction of $G$ to $V$. As shown 
above, $V\left(T_{w_{1}}\right) \cap V\left(T_{w_{2}}\right)=\emptyset$. Since $G$ has no even closed walks of length $>4$, it follows that there is for each $v \in V\left(T_{w}\right)$ a unique walk, whose edges are all distinct from one another, and which connects $v$ with $w$. In fact, if there exist two such walks connecting $v$ with $w$, say $P_{1}, P_{2}$, then their lengths must be both odd or both even, since $G$ is bipartite. By Theorem 1.3, the lengths of $P_{1}$ and $P_{2}$ are both 2 . In fact, since $G$ is Freiman, there exist no primitive even walks in $G$ of length $>4$, thus the lengths of $P_{1}$ and $P_{2}$ are both 1 or 2 . If their lengths are both 1 , then $G$ have a multiple edge connecting $v$ with $w$. But the graph $G$ is simple without multiple edge, thus the union of $P_{1}$ and $P_{2}$ is a 4-cycle of $G$. This implies $v \in V(H)$, a contradiction. Now the property that for each $v \in V\left(T_{w}\right)$ there exists a unique walk connecting $v$ with $w$, implies that $T_{w}$ is a tree.

A typical example of a bipartite Freiman graph is shown in the next figure.

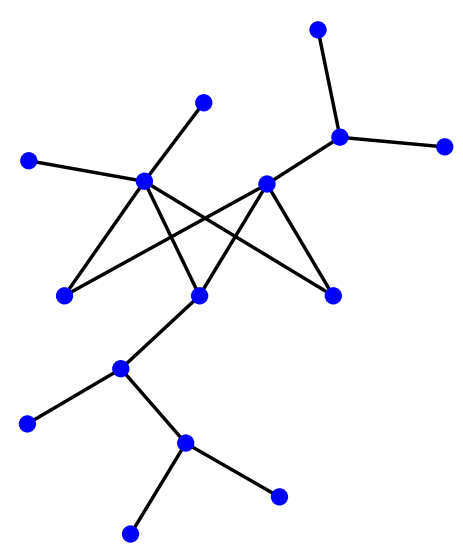

Figure 1

2.2. Cycle matroids. Given a finite simple graph $G$ on $[n]$. The cycle matroid of $G$ is the matroid whose ground set is $E(G)$, and whose bases are the sets $E(F)$ with $F$ a spanning forest of $G$. A spanning forest of $G$ is a maximal acyclic subgraph of $G$.

A matroid $M$ is called graphic, if for some simple graph $G, M$ is isomorphic to the cycle graph of $G$.

Let $K$ be a field. Let $M$ be a matroid on the ground set $[m]$, and $\mathcal{B}$ the set of its bases. To $M$ we attach the ideal $I_{M} \subset S=K\left[x_{1}, \ldots, x_{m}\right]$, whose generators are the monomials $u_{B}$ with $u_{B}=\prod_{i \in B} x_{i}$ and $B \in \mathcal{B}$. The ideal $I_{M}$ is called the matroidal ideal of $M$. The fiber cone of $I_{M}$ is the base ring of $M$. We say that $M$ is a Freiman matroid, if $I_{M}$ is a Freiman ideal.

Let $E(G)=\left\{e_{1}, \ldots, e_{m}\right\}$, and $M$ the cycle matroid of $G$. Then $I_{M} \subset K\left[x_{1}, \ldots, x_{m}\right]$ is the ideal generated by the monomials $u_{F}=\prod_{e_{i} \in F} x_{i}$ with $F$ a spanning forest of $G$.

As the main result of this subsection we have 
Theorem 2.5. Let $M$ be the cycle matroid of $G$. Then the following conditions are equivalent:

(a) $M$ is a Freiman matroid.

(b) $G$ contains at most one cycle.

(c) $F\left(I_{M}\right)$ is a polynomial ring.

We will need the following lemmas before proving Theorem 2.5. First, we have

Lemma 2.6. Let $G$ be a finite simple graph whose cycle matroid is $M$. Let $E$ be a subset of the edges of $G, \Gamma$ the graph with $E(\Gamma)=E$ and $N$ be the cycle matroid of $\Gamma$. Then $N$ is a Freiman matroid.

Proof. We observe that $F\left(I_{N}\right)$ is a combinatorial pure subring of $F\left(I_{M}\right)$ in the sense of [15]. We can write $F\left(I_{M}\right)=B / J$ and $F\left(I_{N}\right)=A / I$, where $A$ and $B$ are polynomial rings and $J \subset B$ and $I \subset A$ are the binomial relation ideals of $F\left(I_{M}\right)$ and $F\left(I_{N}\right)$, respectively. By [15, Corollary 2.5] we have $\beta_{i j}^{A}(I) \leq \beta_{i j}^{B}(J)$ for all $i, j$. Since $M$ is a Freiman matroid, it follows from Theorem 1.3 that $J$ has a 2-linear $B$-resolution. Therefore the above inequality of Betti numbers implies that $I$ has a 2-linear $A$-resolution. Furthermore, since $F\left(I_{N}\right)$ is normal, $F\left(I_{N}\right)$ is CohenMacaulay, by Hochster's theorem, see [14]. We now apply again Theorem 1.3 and conclude that $N$ is a Freiman matroid.

Lemma 2.7. Let $G$ be a finite simple graph consisting of two cycles $C_{1}$ and $C_{2}$ which have at most one vertex in common. Then the cycle matroid of $G$ is not a Freiman matroid.

Proof. Let $M_{i}$ be the cycle matroid of $C_{i}$, and let $r_{i}=\left|E\left(C_{i}\right)\right|$. Then $I_{M_{i}}$ can be identified with the squarefree Veronese ideal $I_{r_{i}, r_{i}-1}$ of degree $r_{i}-1$ in $r_{i}$ indeterminates. Hence the fiber cone of $I_{M_{i}}$ is isomorphic to $K\left[I_{r_{i}, r_{i}-1}\right]$ and this ring is isomorphic to a polynomial ring $S_{i}$ over $K$ in $r_{i}$ indeterminates. Since $C_{1}$ and $C_{2}$ have at most one vertex in common, each spanning forest of $G$ is obtained from $G$ by removing one edge from $C_{1}$ and another edge from $C_{2}$. This shows that $I_{M}=I_{M_{1}} I_{M_{2}}$. Hence the fiber cone of $I_{M}$ is isomorphic to the Segre product $T=S_{1} \# S_{2}$ of the polynomial rings $S_{1}$ and $S_{2}$.

By [8, Theorem 4.2.3] we know that $\operatorname{dim} T=r_{1}+r_{2}-1$. Thus $\operatorname{Hilb}_{T}(t)=$ $h(T ; t) /(1-t)^{r_{1}+r_{2}-1}$, and so the $a$-invariant $a(T)$ of $T$ is equal to $\operatorname{deg} h(T ; t)-\left(r_{1}+\right.$ $r_{2}-1$ ) (see [3, Definition 4.4.4]). Hence we get

$$
\operatorname{deg} h(T ; t)(t)=a(T)+\left(r_{1}+r_{2}-1\right) .
$$

On the other hand, if $\omega_{T}$ is the canonical module of $T$, then

$$
a(T)=-\min \left\{i:\left(\omega_{T}\right)_{i} \neq 0\right\},
$$

see [8, Definition 3.1.4]. We now use another result of Goto and Watanabe [8, Theorem 4.2.3] which says that $\omega_{T} \cong \omega_{S_{1}} \# \omega_{S_{2}}$. Since $\omega_{S_{i}}=S_{i}\left(-r_{i}\right)$, we have $\omega_{T} \cong S_{1}\left(-r_{1}\right) \# S_{2}\left(-r_{2}\right)$. The $i$ th graded component $\left(\omega_{T}\right)_{i}$ of $\omega_{T}$ is isomorphic to

$$
\left(S_{1}\left(-r_{1}\right) \# S_{2}\left(-r_{2}\right)\right)_{i}=\left(S_{1}\right)_{i-r_{1}} \otimes\left(S_{2}\right)_{i-r_{2}} .
$$


Therefore, $\min \left\{i:\left(\omega_{T}\right)_{i} \neq 0\right\}=\max \left\{r_{1}, r_{2}\right\}$. Combining (2) with (3i), we get

$$
\operatorname{deg} h(T ; t)=r_{1}+r_{2}-1-\max \left\{r_{1}, r_{2}\right\} .
$$

Since each cycle has at least 3 edges, we see that $r_{i} \geq 3$. It follows that $\operatorname{deg} h(T ; t) \geq$ 2. Thus Theorem 1.3 implies that $M$ is not a Freiman matroid.

In the case that $C_{1}$ and $C_{2}$ are connected by a walk $P$, we have $I_{M}=u I_{M_{1}} I_{M_{2}}$, where $u$ is the monomial whose factors correspond to the edge of $P$. Then $K\left[I_{M}\right]=$ $K\left[u I_{M_{1}} I_{M_{2}}\right] \cong K\left[I_{M_{1}} I_{M_{2}}\right]$, and the rest of the proof is as before.

Lemma 2.8. Let $G$ be a finite simple graph consisting of a cycle $C$ of length $r$ and a walk $W$ of length $s$ which connects two non-consecutive vertices of $C$. Then the cycle matroid $M$ of $G$ is not a Freiman matroid.

Proof. By hypothesis, the walk $W$ divides cycle $C$ into two walks $W_{1}$ and $W_{2}$, where $W_{1}, W_{2}$ and $W$ have common starting point and endpoint. Thus $C=W_{1} \cup W_{2}$. For simplicity we set $W_{3}=W$. Let $M_{i}$ be the cycle matroid of $W_{i}, r_{i}=\left|E\left(W_{i}\right)\right|$, and let $M_{4}$ be the cycle matroid of $C$ and $r_{4}=|E(C)|$. Then $r_{3}=s, r_{4}=r_{1}+r_{2}=r$. By the proof of Lemma 2.7, the fiber cone $F\left(I_{M_{i}}\right)$ of $I_{M_{i}}$ is isomorphic to $K\left[I_{r_{i}, r_{i}-1}\right]$ and this ring is isomorphic to a polynomial ring $S_{i}$ over $K$ in $r_{i}$ indeterminates. Therefore, we get $I_{M}=I_{r_{3}, r_{3}-1} I_{r_{1}+r_{2}, r_{1}+r_{2}-1}+J I_{r_{3}, r_{3}}$, where $J=I_{r_{1}, r_{1}-1} I_{r_{2}, r_{2}-1}$. We have $I_{r_{3}, r_{3}} \subset I_{r_{3}, r_{3}-1}$ and $I_{r_{1}+r_{2}, r_{1}+r_{2}-1} \subset J$, Let $S=S_{3} \otimes S_{4}$ and $\operatorname{Hilb}_{F\left(I_{M}\right)}(t)=$ $h(S ; t) /(1-t)^{\ell}$, where $\ell=\operatorname{dim}\left(F\left(I_{M}\right)\right)$. By [13, Theorem 1.6], we have

$$
\begin{aligned}
\operatorname{Hilb}_{S / I_{M}}(t) & =\operatorname{Hilb}_{S /\left(I_{r_{3}, r_{3}-1} I_{r_{1}+r_{2}, r_{1}+r_{2}-1}+J I_{r_{3}, r_{3}}\right)}(t) \\
& =\operatorname{Hilb}_{S_{3} / I_{r_{3}, r_{3}-1}}(t) \operatorname{Hilb}_{I_{r_{1}+r_{2}, r_{1}+r_{2}-1}}(t)+\operatorname{Hilb}_{S_{4} / J}(t) \operatorname{Hilb}_{I_{r_{3}, r_{3}}}(t) \\
& +\operatorname{Hilb}_{S_{3} / I_{r_{3}, r_{3}}}(t) \operatorname{Hilb}_{S_{4} / I_{r_{1}+r_{2}, r_{1}+r_{2}-1}}(t) \\
& =\operatorname{Hilb}_{I_{r_{1}+r_{2}, r_{1}+r_{2}-1}}(t)+\operatorname{Hilb}_{S_{4} / J}(t) \operatorname{Hilb}_{I_{r_{3}, r_{3}}}(t)+\operatorname{Hilb}_{S_{3} / I_{r_{3}, r_{3}}}(t) .
\end{aligned}
$$

Hence

$$
\begin{aligned}
\operatorname{Hilb}_{F\left(I_{M}\right)}(t) & =\frac{1}{(1-t)^{r_{3}+r_{4}}}-\operatorname{Hilb}_{S / I_{M}}(t) \\
& =\frac{1}{(1-t)^{r_{3}+r_{4}}}-\left[\sum_{m=0}^{\infty}\left(\begin{array}{c}
m+r_{4}-1 \\
m
\end{array}\right) t^{m}+\left(\frac{1}{(1-t)^{r_{4}}}-\frac{h\left(S_{4} ; t\right)}{(1-t)^{r_{4}-1}}\right) \sum_{m=0}^{\infty} t^{m}\right. \\
& \left.+\frac{1}{(1-t)^{r_{3}-1}}\right] \\
& =\frac{1}{(1-t)^{r_{3}+r_{4}}}-\left[\frac{1}{(1-t)^{r_{4}}}+\left[\frac{1}{(1-t)^{r_{4}}}-\frac{h\left(S_{4} ; t\right)}{(1-t)^{r_{4}-1}}\right] \frac{1}{1-t}+\frac{1}{(1-t)^{r_{3}-1}}\right] \\
& =\frac{1}{(1-t)^{r_{3}+r_{4}}}-\frac{(1-t)^{r_{3}}+(1-t)^{r_{3}-1}\left[1-(1-t) h\left(S_{4} ; t\right)\right]+(1-t)^{r_{4}+1}}{(1-t)^{r_{3}+r_{4}}} \\
& =\frac{1-(1-t)^{r_{3}}-(1-t)^{r_{3}-1}\left[1-(1-t) h\left(S_{4} ; t\right)\right]-(1-t)^{r_{4}+1}}{(1-t)^{r_{3}+r_{4}}} \\
& =\frac{1-(1-t)^{s}-(1-t)^{s-1}\left[1-(1-t) h\left(S_{4} ; t\right)\right]-(1-t)^{r+1}}{(1-t)^{r+s}},
\end{aligned}
$$


where $\operatorname{deg} h\left(S_{4} ; t\right)=\min \left\{r_{1}, r_{2}\right\}-1$, as seen in the proof of Lemma 2.7 .

We consider the following two cases:

(1) If $s=1$, then $\operatorname{Hilb}_{F\left(I_{M}\right)}(t)=\frac{h\left(S_{4} ; t\right)-1-(1-t)^{r}}{(1-t)^{r}}$ and $h(S ; t)=h\left(S_{4} ; t\right)-1-(1-t)^{r}$, Since $\operatorname{deg} h\left(S_{4} ; t\right)=\min \left\{r_{1}, r_{2}\right\}-1$ and $r=r_{1}+r_{2}, \operatorname{deg} h(S ; t) \geq \max \left\{r_{1}, r_{2}\right\}+1$. Notice that $r_{i} \geq 2$, we have that $\operatorname{deg} h(S ; t) \geq 3$;

(2) If $s \geq 2$, then $h(S ; t)=1-(1-t)^{s}-(1-t)^{s-1}\left[1-(1-t) h\left(S_{4} ; t\right)\right]-(1-t)^{r+1}$ such that $h(S ; 1)=1$ and $\operatorname{deg} h(S ; t) \geq 5$.

Thus Theorem 1.3 implies that the cycle matroid $M$ of $G$ is not a Freiman matroid.

Now we are ready to prove Theorem 2.5.

Proof. (a) $\Rightarrow(\mathrm{b})$ : Suppose there is a cycle $C$ for which there exists a walk $W$ which connects two non-consecutive vertices of $C$. Then let $\Gamma$ be the graph with edges $E(W) \cup E(C)$. By Lemma 2.8 the cycle matroid of $\Gamma$ is not a Freiman matroid, and so $M$ is not a Freiman matroid by Lemma 2.6, a contradiction. Thus there is no cycle $C$ of $G$ such that there exists a walk $W$ which connects two non-consecutive vertices of $C$.

Let us now assume that $G$ contains at least two cycles $C_{1}$ and $C_{2}$. We discuss two cases: (i) $V\left(C_{1}\right) \cap V\left(C_{2}\right)=\emptyset$, and (ii) $V\left(C_{1}\right) \cap V\left(C_{2}\right) \neq \emptyset$.

Let $\Gamma$ be the graph with $E(\Gamma)=E\left(C_{1}\right) \cup E\left(C_{2}\right)$. In case (i), the cycle matroid of $\Gamma$ is not a Freiman matroid, see Lemma 2.7. Therefore, according to Lemma 2.6. also $M$ is not a Freiman matroid.

In case (ii), by using Lemma 2.6, we may assume that $\Gamma=G$. Let $S=V\left(C_{1}\right) \cap$ $V\left(C_{2}\right)$, and $\Sigma$ the graph which is obtained from $G$ by restriction to $S$. Then $\Sigma$ is a disjoint union of walks and some isolated vertices. If $\Sigma$ is just a single vertex, $M$ is not a Freiman matroid by Lemma 2.7, and if $\Sigma$ is just a single walk, then the edges $E\left(C_{1}\right) \cup E\left(C_{2}\right) \backslash E(\Sigma)$ form a cycle $C$ and $\Sigma$ is a walk which connects two non-consecutive vertices of $C$. Thus Lemma 2.8 implies that $M$ is not a Freiman matroid. Finally assume that $\Sigma$ has more than one connected component. Let $V\left(C_{1}\right)=\left\{v_{1}, \ldots, v_{r}\right\}$ and $V\left(C_{2}\right)=\left\{w_{1}, \ldots, w_{s}\right\}$ with a counter-clockwise labeling of the vertices. We may assume without loss of generality that $v_{1}=w_{1}$ and $v_{2} \neq w_{2}$, since $\Sigma$ is not connected. There exist integers $a, b$ with the property that $v_{a}=w_{b}$ and $v_{i} \neq w_{j}$ for all $i, j$ with $1<i<a$ and $1<j<b$. It then follows that the walk $W^{\prime}$ with $V\left(W^{\prime}\right)=\left\{w_{1}, w_{2}, \ldots, w_{b}\right\}$ is a walk connecting the vertices $v_{1}$ and $v_{a}$ of $C_{1}$. Thus $M$ is not a Freiman matroid.

(b) $\Rightarrow$ (c): Let $G_{1}, \ldots, G_{s}$ be the connected components of $G$ and let $M_{i}$ be the cycle matroid of $G_{i}$. Since $G$ contains at most one cycle, we may assume that the components $G_{i}$ are trees for $i \geq 2$. Then, as in the proof of Lemma 2.8, we see that $I_{M_{1}}=u I_{r, r-1}$ if $G_{1}$ contains a cycle $C$ with $|E(C)|=r$, or otherwise $I_{M_{1}}$ is a principal monomial ideal. Moreover, all $I_{M_{i}}$ for $i \geq 2$ are principal monomial ideals. Since $I_{M}=I_{M_{1}} \cdots I_{M_{s}}$, we have $K\left[I_{M}\right] \cong K\left[I_{M_{1}}\right]$, and hence $K\left[I_{M}\right]$ is a polynomial ring.

(c) $\Rightarrow($ a) is obvious. 
We close this paper with discussing the regularity of the base ring of the cycle matroid of a simple graph. Let, as before, $G$ be a finite simple graph on $[n]$ and $E(G)=\left\{e_{1}, \ldots, e_{m}\right\}$ its edge set. Let $\mathbf{e}_{1}, \ldots, \mathbf{e}_{m}$ denote the canonical coordinate vectors of $\mathbb{R}^{m}$. Given a subset $F \subset E(G)$, one defines $\rho(F)=\sum_{e_{i} \in F} \mathbf{e}_{i} \in \mathbb{R}^{m}$. For example, $\rho\left(\left\{e_{2}, e_{3}, e_{5}\right\}\right)=\mathbf{e}_{2}+\mathbf{e}_{3}+\mathbf{e}_{5}=(0,1,1,0,1,0, \ldots, 0) \in \mathbb{R}^{m}$. The base polytope $\mathcal{P}(M)$ of the cycle matroid $M$ of $G$ is the convex polytope which is the convex hull of the finite set $\{\rho(F): \rho(F) \in \mathcal{B}\}$ in $\mathbb{R}^{m}$. One has $\operatorname{dim} F\left(I_{M}\right)=$ $\operatorname{dim} \mathcal{P}(M)+1$.

Recall that, when $G$ is connected, a vertex $i \in[n]$ of $G$ is said to be a cut vertex if the induced subgraph $\left.G\right|_{[n] \backslash\{i\}}$ of $G$ on $[n] \backslash\{i\}$ is disconnected. A finite connected simple graph $G$ is called 2-connected if no vertex of $G$ is a cut vertex of $G$. It then follows from [20, Theorem 5] that

Lemma 2.9. Let $G$ be a finite 2 -connected simple graph on $[n]$ with e edges. Let $M$ be the cycle matroid of $G$ and $\mathcal{P}(M)$ the base polytope of $M$. Then

$$
\ell\left(I_{M}\right)=\operatorname{dim} \mathcal{P}(M)+1=e .
$$

Corollary 2.10. Let $G$ be a finite connected simple graph on $[n]$ with e edges and write $c$ for the number of cut vertices of $G$. Let $M$ be the cycle matroid of $G$ and $\mathcal{P}(M)$ the base polytope of $M$. Then

$$
\ell\left(I_{M}\right)=\operatorname{dim} \mathcal{P}(M)+1=e-c .
$$

Proof. Let, say, $n \in[n]$ be a cut vertex of $G$. Let $G^{\prime}$ and $G^{\prime \prime}$ be the connected components of the induced subgraph $\left.G\right|_{[n-1]}$. Write $U$ for the vertex set of $G^{\prime}$ and $V$ that of $G^{\prime \prime}$. Let $M^{\prime}$ be the cycle matroid of $\left.G\right|_{U \cup\{n\}}$ and $M^{\prime \prime}$ that of $\left.G\right|_{V \cup\{n\}}$. Let $e^{\prime}$ be the number of edges of $\left.G\right|_{U \cup\{n\}}$ and $e^{\prime \prime}$ that of $\left.G\right|_{V \cup\{n\}}$. Since the base ring $F\left(I_{M}\right)$ is the Segre product of $F\left(I_{M^{\prime}}\right)$ and $F\left(I_{M^{\prime \prime}}\right)$, it follows that $\operatorname{dim} F\left(I_{M}\right)=$ $\operatorname{dim} F\left(I_{M^{\prime}}\right)+\operatorname{dim} F\left(I_{M^{\prime \prime}}\right)-1$. Let $c^{\prime}$ be the number of cut vertices of $G^{\prime}$ and $c^{\prime \prime}$ that of $G^{\prime \prime}$. Then using induction yields $\operatorname{dim} F\left(I_{M^{\prime}}\right)=e^{\prime}-c^{\prime}$ and $\operatorname{dim} F\left(I_{M^{\prime \prime}}\right)=e^{\prime \prime}-c^{\prime \prime}$. Hence $\operatorname{dim} F\left(I_{M}\right)=\left(e^{\prime}-c^{\prime}\right)+\left(e^{\prime \prime}-c^{\prime \prime}\right)-1$. Since $e=e^{\prime}+e^{\prime \prime}$ and $c=c^{\prime}+c^{\prime \prime}+1$, the desired formula $\operatorname{dim} F\left(I_{M}\right)=\operatorname{dim} \mathcal{P}(M)+1=e-c$ follows.

Corollary 2.11. Let $G$ be a finite disconnected simple graph on $[n]$ with e edges and $G_{1}, \ldots, G_{s}$ its connected component. Let $c_{i}$ denote the number of cut vertices of $G_{i}$ for $1 \leq i \leq s$. Let $M$ be the cycle matroid of $G$ and $\mathcal{P}(M)$ the base polytope of $M$. Then

$$
\ell\left(I_{M}\right)=\operatorname{dim} \mathcal{P}(M)+1=e-c-s+1 .
$$

Proof. Let $M_{i}$ be the cycle matroid of $G_{i}$ for $1 \leq i \leq s$. Since the base $\operatorname{ring} F\left(I_{M}\right)$ is the Segre product of the base rings $F\left(I_{M_{1}}\right), \ldots, F\left(I_{M_{s}}\right)$, the required formula follows immediately from Corollary 2.10.

Let $G$ be a finite 2-connected simple graph on $[n]$ with $e$ edges and $\mathcal{P}(M)$ the base polytope of the cycle matroid $M$ of $G$. Thus $\operatorname{dim} \mathcal{P}(M)=e-1$. We introduce the sequence of integers $\left(\delta_{0}, \delta_{1}, \delta_{2}, \ldots\right)$ by the formula

$$
(1-\lambda)^{e} \sum_{i=0}^{\infty} \delta_{i} t^{i}=\sum_{\substack{i=0 \\ 14}}^{\infty}\left|i \mathcal{P}(M) \cap \mathbb{Z}^{m}\right| t^{i}
$$


where $i \mathcal{P}(M)$ is the $i$ th dilated polytope $\{i \alpha: \alpha \in \mathcal{P}(M)\}$ of $\mathcal{P}(M)$. It then follows that $\delta_{i}=0$ for $i>\operatorname{dim} \mathcal{P}(M)=e-1$. We say that $\delta(\mathcal{P}(M))=\left(\delta_{0}, \delta_{1}, \ldots, \delta_{e-1}\right)$ is the $\delta$-vector of $\mathcal{P}(M)$ and $\delta(\mathcal{P}(M) ; t)=\sum_{i=0}^{e-1} \delta_{i} t^{i}$ is the $\delta$-polynomial of $\mathcal{P}(M)$. Furthermore, introducing the integer $r_{0} \geq 1$ by

$$
r_{0}=\min \left\{r \in \mathbb{Z}: r \geq 1 \text { and } r(\mathcal{P}(M) \backslash \partial \mathcal{P}(M)) \cap \mathbb{Z}^{m} \neq \emptyset\right\},
$$

where $\mathcal{P}(M) \backslash \partial \mathcal{P}(M)$ is the relative interior of $\mathcal{P}(M)$, one has the formula

$$
\operatorname{deg} \delta(\mathcal{P}(M) ; t)=e-r_{0} .
$$

We refer the reader to 12 for the detailed information about $\delta$-vectors.

Since the base polytope $\mathcal{P}(M)$ possesses the integer decomposition property $(9$, p. 250]), it follows that

Lemma 2.12. The $\delta$-polynomial $\delta(\mathcal{P}(M) ; t)$ of $\mathcal{P}(M)$ coincides with the h-polynomial of $F\left(I_{M}\right)$.

Since the base ring $F\left(I_{M}\right)$ is Cohen-Macaulay, it is well-known that the regularity $\operatorname{reg}\left(F\left(I_{M}\right)\right)$ of $F\left(I_{M}\right)$ is one more than the degree of the $h$-polynomial $h\left(F\left(I_{M}\right) ; t\right)$ of $F\left(I_{M}\right)$. In other words, the regularity $\operatorname{reg}\left(F\left(I_{M}\right)\right)$ is one more than the degree of the $\delta$-polynomial $\delta(\mathcal{P}(M) ; t)$ of $\mathcal{P}(M)$. Since the hyperplanes $x_{i}=0$ and $x_{i}=1$ of $\mathbb{R}^{m}$ are supporting hyperplanes of $\mathcal{P}(M)$ for $1 \leq i \leq n$, it follows that no lattice point of $\mathbb{R}^{m}$ belongs to the relative interior of $\mathcal{P}(M)$. Hence, by using the formula (4), one has $\left.\operatorname{deg} \delta(\mathcal{P}(M) ; t)=\operatorname{deg} h\left(F\left(I_{M}\right)\right) ; t\right) \leq e-2$. Thus, in particular $\operatorname{reg}\left(F\left(I_{M}\right)\right) \leq e-1$. Furthermore, Theorem 2.5 guarantees that, unless $F\left(I_{M}\right)$ is the polynomial ring, one has $\operatorname{deg} h\left(F\left(I_{M}\right), t\right) \geq 2$ and $\operatorname{reg}\left(I_{M}\right) \geq 3$. As a result,

Theorem 2.13. Let $G$ be a finite 2-connected simple graph on $[n]$ with e edges. Let $M$ be the cycle matroid of $G$. Unless $F\left(I_{M}\right)$ is the polynomial ring, one has

$$
3 \leq \operatorname{reg}\left(F\left(I_{M}\right)\right) \leq e-1 .
$$

It is natural to ask, given integers $3 \leq r<e$, if there exists a 2-connected simple graph $G$ with $e$ edges for which $\operatorname{reg}\left(F\left(I_{M}\right)\right)=r$, where $M$ is the cycle matroid of $G$ and $F\left(I_{M}\right)$ is the base ring of $M$.

Corollary 2.14. Let $G$ be a finite disconnected simple graph on $[n]$ with e edges and $G_{1}, \ldots, G_{s}$ its connected components. Let $c_{i}$ denote the number of cut vertices of $G_{i}$ for $1 \leq i \leq s$. Let $M$ be the cycle matroid of $G$. Unless $F\left(I_{M}\right)$ is the polynomial ring, one has

$$
3 \leq \operatorname{reg}\left(F\left(I_{M}\right)\right) \leq e-c-s .
$$

Finally, since the toric ideal of the base ring of a cycle matroid of a finite simple graph is generated by quadratic binomials [2], it follows that no base ring of a cycle matroid has a linear resolution.

Acknowledgement. This paper is supported by the National Natural Science Foundation of China (11271275) and by the Foundation of the Priority Academic Program Development of Jiangsu Higher Education Institutions. 


\section{REFERENCES}

[1] S. S. Abhyankar, Local rings of high embedding dimension, Amer. J. Math. 89 (1967), 10731077.

[2] J. Blasiak, The toric ideal of a graphic matroid is generated by quadrics, Combinatorica 28 (2008), 283-297.

[3] W. Bruns and J. Herzog, Cohen-Macaulay rings, Cambridge University Press, Cambridge, UK, 1998.

[4] K. J. Böröczky, F. Santos and O. Serra, On sumsets and convex hull, Discrete Comput. Geom. 52 (2014), 705-729.

[5] D. Eisenbud and S. Goto, Linear free resolutions and minimal multiplicity, J. Algebra 88 (1984), 89-133.

[6] J. A. Eagon and D. G. Northcott, Ideals defined by matrices and a certain complex associated with them, Proc. R. Soc. Lond. Ser. A 269 (1962), 188-204.

[7] G. A. Freiman, Foundations of a structural theory of set addition, Translations of mathematical monographs 37, Amer. Math. Soc. Providence, Phode Island, 1973.

[8] S. Goto, K. Watanabe, On graded rings, I, J. Math. Soc. Japan 30 (1978), 179-213.

[9] J. Herzog and T. Hibi, Monomial ideals, Graduate Texts in Mathematics 260, Springer, London, 2010.

[10] J. Herzog, M. Mohammadi Saem and N. Zamani, On the number of generators of powers of an ideal, arXiv: 1707.07302v1.

[11] J. Herzog and G. J. Zhu, Freiman ideals, arXiv: 1709.02827v1.

[12] T. Hibi, "Algebraic Combinatorics on Convex Polytopes," Carslaw Publications, Glebe, N. S. W., Australia, 1992.

[13] L. T. Hoa and N. D. Tam, On some invariants of a mixed product of ideals, Arch. Math. 94 (2010), 327-337.

[14] M. Hochster, Rings of invariants of tori, Cohen-Macaulay rings generated by monomials, and polytopes, Ann. of Math. 96 (1972), 228-235.

[15] H. Ohsugi, J. Herzog and T. Hibi, Combinatorial pure subrings, Osaka J. Math. 37 (2000), $745-757$.

[16] H. Ohsugi and T. Hibi, Toric Ideals generated by Quadratic Binomials, J. Algebra 218 (1999), 509-527.

[17] J. D. Sally, On the associated graded rings of a local Cohen-Macaulay ring, J. Math. Kyoto Univ. 17 (1977), 19-21.

[18] K. Shah, On the Cohen-Macaulayness of the fiber cone of an ideal, J. Algebra 143 (1991), $156-172$.

[19] Y. V. Stanchescu, On the simplest inverse problem for sums of sets in several dimensions, Combinatorica 18 (1998), 139-149.

[20] N. L. White, The basis monomial ring of a matroid, Advances in Math. 24 (1977), 292-297.

Jürgen Herzog, Fachbereich Mathematik, Universität Duisburg-Essen, Campus Essen, 45117 Essen, Germany

E-mail address: juergen.herzog@uni-essen.de

Takayuki Hibi, Department of Pure and Applied Mathematics, Graduate School of Information Science and Technology, Osaka University, Toyonaka, Osaka 5600043, JAPAN

E-mail address: hibi@math.sci.osaka-u.ac.jp

Guanguun Zhu, School of Mathematical Sciences, Soochow University, Suzhou 215006, P. R. CHINA

E-mail address: zhuguangjun@suda.edu.cn 